\title{
ACTUACIONES, PROGRAMAS Y ACTIVIDADES DESARROLLADAS DESDE EL DEPARTAMENTO DE ORIENTACIÓN: Análisis de una experiencia en el IES TORREBLANCA de Sevilla durante el curso escolar 1998/99
}

\author{
PROGRAMAS AND ACTIVITIES IMPLEMENT BY THE \\ GUIDANCE DEPARTMENT:
}

Analysis of a practical experience carried out at the Iestorreblanca (High School) in Seville during the academic year 1998/99

\author{
Manuel Moreno* \\ I.E.S. Isbilya
}

\begin{abstract}
RESUMEN
Con el presente trabajo se pretende hacer un análisis y valoración detallada de lo que ha supuesto la dinamización desde el Departamento de Orientación, el diseño, puesta en práctica y evaluación de las diferentes actuaciones, programas y actividades en los distintos ámbitos de orientación (personal, escolar y vocacional-profesional) con los diversos agentes de la comunidad educativa; así como las distintas medidas de atención a la diversidad para nuestro alumnado (Adaptaciones curriculares, Programa de apoyo a la Integración, Diversificación Curricular y Acción Tutorial).
\end{abstract}

Palabras clave: Departamento de Orientación, Atención a la Diversidad, Acción tutorial, Orientación Vocacional, Comunidad Educativa.

\begin{abstract}
In this projet we intend to analyse and evaluate in detail the incidence of the activities proposed by the Guidance Department: the design, development and evaluation of several programmes and activities in the different fields of guidance (personal, academic and vocational) and their relation with the different agents of the educational community. Besides that, we explore the different ways to deal with the diversity of our pupils (curricular adaptations, integration programmes, curricular diversification and Pastoral Care).
\end{abstract}

* Licenciado en Ciencias de la Educación y Diplomado en Profesorado de E.G.B. (Lengua Española e Idiomas). En la actualidad trabaja como Orientador Escolar. 
Key words: Guidance Department, Atention to Diversity, Pastoral Care, Vocational Training, Educational Community.

\section{Introducción}

Con el presente trabajo, se pretende dar una doble visión desde la práctica orientadora que iniciamos desde el curso académico 1992.

Por un lado hacer referencia a las actuaciones y actividades en los diferentes ámbitos que se han venido desarrollando desde el Departamento de Orientación. Las estrategias de dinamización y coordinación por parte de la Jefatura de este Departamento con los distintos agentes de la comunidad educativa han hecho posible la planificación, puesta en practica y evaluación de los diferentes procesos y programas de intervención.

Por otro lado, la orientación realizada al alumnado, profesorado y familias así como la coordinación mantenida con el Equipo Directivo, especialmente las Jefaturas de Estudios (Secundaria Obligatoria, adjuntas al Bachillerato y F.P. Específica) y la relación que se mantuvo con el entorno a lo largo del curso escolar 1998/99.

El Departamento de Orientación, no deja de ser un órgano de apoyo dentro de nuestro contexto general del centro, desde donde se asume formalmente la tarea de impulsar y promover las actividades de tutoría y orientación del mismo con el fin de asesorar la dimensión orientadora inherente a la educación y a la función docente.

La existencia del Departamento se rentabiliza en la medida que se van encontrando respuestas a nuestros planteamientos y en la medida que los agentes implicados (alumnos/as, familias y profesorado) participan y colaboran a lo largo de todo el proceso.

\section{Contextualización de la experiencia}

El IES TORREBLANCA es un Instituto de Educación Secundaria que cuenta con una matricula de 80 alumnos/as aproximadamente. Concebido como un centro de Formación Profesional ha vivido la transformación del sistema educativo (Reforma de las Enseñanzas Medias, anticipación, experimentación y desarrollo de la actual Ley de Ordenación del Sistema Educativo desde sus comienzos en Andalucía).

Actualmente se imparten dos etapas educativas. Por un lado, la Educación Secundaria Obligatoria con los dos ciclos, donde se integra alumnado con necesidades educativas especiales permanentes a lo largo de dicha etapa. Dentro de la Educación Secundaria Postobligatoria se imparten bachilleratos LOGSE (11 y 21) en las modalidades de Humanidades y Ciencias Sociales, Ciencias de la Naturaleza y de la Salud y Tecnológico con todos sus itinerarios correspondientes; cuenta además con dos Programas de Garantía social (Electricidad aplicada al automóvil y Servicios Auxiliares de Oficina); dentro de la Formación Profesional Específica se imparten ciclos Formativos de Grado Medio (Carrocería, Gestión Administrativa, Estética y Peluquería); en los Ciclos Formativos de Grado Superior las titulaciones que se imparten son las de Secretariado, Automoción y Estética.

El Instituto se encuadra en un barrio hoy dentro del municipio de Sevilla con unas marcadas características de marginalidad de un sector de su población y un nivel de vida me- 
dio-bajo en sus habitantes. Es, en definitiva, un barrio de trabajadores aunque con problemas de delincuencia y drogadicción propios de los barrios marginales de las grandes urbes en el sector citado anteriormente.

Por otra parte, la plantilla de profesores/as que conforma el centro es definitiva en un $95 \%$ del total del profesorado que imparte clases y la media de edad se sitúa en torno a los 40 años de edad, viviendo la gran mayoría del profesorado la anticipación, experimentación y desarrollo del nuevo sistema educativo.

\section{Actividades realizadas con los alumnos/as}

De la planificación, implementación y evaluación de los programas previstos en el Plan de Acción tutorial y Orientación, podemos valorar los siguientes resultados:

\section{Actividades grupales}

Realización de actividades comunes a todos los niveles dentro de la Educación Secundaria Obligatoria: normas de convivencia, Derechos y Deberes de los alumnos, preparación de las distintas evaluaciones, elección de delegados, estudio dirigido, revisión del rendimiento académico del alumnado, dinámicas grupales, ...

En el Primer Ciclo de E.S.O. y, según las necesidades de la tutoría se desarrollaron los temas, además de los anteriores citados:

- Mejora de la autoestima.

- Mejora de la comunicación, la convivencia y el clima de trabajo en el grupo.

- Técnicas de estudio. Los distintos procedimientos y técnicas específicas se van integrando paulatinamente de un modo contextualizado en las distintas áreas y materias del currículum en cada uno de los niveles de la ESO, sin llegar a considerar las técnicas de trabajo intelectual como algo puntual, aislado o marginal del propio currículum.

- El alcohol: uso y abuso.

- Educación sexual.

- Orientación vocacional e información académica para el próximo curso.

En el SEgundo Ciclo de la ESO se pusieron en práctica además las siguientes actividades y programas:

- Programa de prevención de drogodependencias (8 sesiones en $3^{\circ}$ y $4^{\circ}$ de ESO).

- Educación sexual.

- Orientación Vocacional y Profesional (se ha tratado en profundidad en $4^{\circ}$ de ESO incluido el grupo de alumnos/as que estaban adscritos al programa de Diversificación Curricular).

Los aspectos tratados fueron:

- Conocimiento de sí mismo y del grupo.

- Historial académico del alumnado. 
- Gustos y preferencias profesionales.

- El mundo de los valores.

- Aptitudes.

- El entorno laboral.

- Información académica: $\left(3^{\circ}\right.$ y $4^{\circ}$ de ESO):

- Para $3^{\circ}$ de ESO:

- Materias comunes

- Materias opcionales

- Materias optativas.

- Para $4^{\circ}$ de ESO:

- Los Ciclos Formativos de Grado Medio.

- El Bachillerato.

- Otras alternativas de formación.

- Para $4^{\circ}$ de ESO se ha utilizado, entre otros materiales específicos el programa informatizado ORIENTA y LOGSE, donde se incluían, entre otros contenidos:

- Planes de estudios universitarios.

- Salidas fuera y dentro del Sistema Educativo.

- Salidas profesionales y carreras universitarias: lugares donde se estudia, nota de corte, expectativas laborales, perfil profesionalizador, pasarelas, forma de acceso (modalidad e itinerario desde los Bachilleratos, ...). alto.

El efecto fue bastante satisfactorio, siendo el grado de participación del alumnado muy

- Técnicas de Búsqueda de empleo en colaboración con uno de los profesores del Departamento de Formación y Orientación Laboral durante el tercer trimestre.

- Colaboración de los Departamentos Didácticos y de ramas de F.P. para la realización de mesas redondas por parte del profesorado para los alumnos que iban a estudiar Bachillerato o bien Ciclos Formativos.

EnSEÑANZA POSTOBLIGAToRia: Dentro de esta etapa educativa (Bachilleratos, ciclos formativos de Grado Medio y Superior y Programa de Garantía Social), se valoraron como muy efectivas las siguientes actuaciones:

- Charlas del INEM (Instituto Nacional de empleo) en los C. Formativos de Grado Medio y Superior y Programa de Garantía Social, en la que se tratan los siguientes aspectos:

- Organización y funcionamiento.

- Panorama actual de la contratación.

- Formas de contratación.

- Servicios que ofrece el INEM.

- Actividades para la persona que busca trabajo.

- Técnicas sobre la búsqueda de empleo. 
- Respuesta al guión de interrogantes previamente preparado por el grupo-clase.

- La Fundación FONAT (Centro de Orientación sociolaboral para jóvenes) en horario de tarde y fuera del horario lectivo, imparte para los alumnos del C. Formativo de Grado Medio de Gestión Administrativa, Carrocería y Estética y Peluquería un programa cuyos contenidos versaban sobre la introducción al mundo laboral, características personales, profesionales, instrumentos de búsqueda de empleo, entrevista de selección, contratos de trabajo y pruebas psicotécnicas.

- En Primero de Bachillerato se llevaron a cabo tareas y actividades como:

- Información académica.

- Mesas redondas con la colaboración de los distintos Departamentos Didácticos:

- Materias optativas y específicas y la vinculación con las distintas salidas profesionales y carreras.

- Planes de estudios.

- Programa informatizado LOGSE y ORIENTA.

- Ayuda y asesoramiento individual a los alumnos cuya toma de decisión no estaba definida.

- Preparación de las carpetas-dossier con la información pertinente sobre materias comunes de $2^{\circ}$ de Bachillerato, materias optativas, específicas, itinerarios, planes de estudios, vinculación con las diferentes carreras y ciclos formativos.

- Salidas profesionales, etc.

- En Segundo de Bachillerato además se trabajaron los siguientes contenidos:

- Pruebas de Aptitud para el Acceso a la Universidad: burocracia, días de celebración, ejercicios, notas de corte, lo que debían saber antes de la prueba, durante la prueba y después de la prueba.

- Información sobre los C. Formativos de Grado Superior.

- Planes de estudios universitarios.

- Carpeta-dossier con todos los temas pertinentes.

- Colaboración con el Secretariado de Acceso:

- Facilitar documentación.

- Jornadas sobre el Acceso a la Universidad para el profesorado.

- Charlas para las PAAU (Pruebas de Aptitud para el Acceso a la Universidad).

- Mesas redondas sobre estudios universitarios formada por antiguos alumnos/as del instituto.

- Asesoramiento individual al alumnado.

Todas estas actividades fueron coordinadas por la Jefatura de Estudios de Bachillerato y el Orientador.

\section{Actividades individuales}

El orientador además colabora de modo más individual para: 
- Estudio de casos de alumnos/as con dificultades de aprendizaje, bajo rendimiento escolar, realización evaluación psicopedagógica de alumnado propuesto para ACI o Refuerzo Educativo.

- Derivación previo informe al Centro Audiológico para estudio audiométrico (alumnos con hipoacusia).

- Realización de entrevistas personales individualizadas (ámbito personal, escolar, vocacional o profesional).

- Seguimiento de casos (alumnado con necesidades educativas especiales o Diversificación Curricular).

- Elaboración de evaluaciones psicopedagógicas para alumnos adscritos al Programa de Diversificación Curricular o alumnos con necesidades educativas especiales.

\section{Actividades realizadas con el profesorado.}

Entre las funciones y tareas desempeñadas por el orientador podemos citar entre otras:

- Coordinación con el profesorado para el desarrollo de la Acción tutorial: Profesores de Primer Ciclo de la ESO, $3^{\circ}$ y $4^{\circ}$ de ESO.

- Asesoramiento sobre temas demandados.

- Proporcionar la información, actividades, guiones de trabajo, ... para realizar en horario de tutoría.

- Colaboración con las distintas reuniones de Equipo Docente convocadas por el tutor o la tutora durante el curso escolar.

- Colaboración en la coordinación del proceso evaluador y seguimiento del Programa de Diversificación Curricular.

- Participación y coordinación en el proyecto de trabajo sobre motivación "Maid Marian" (Poyecto europeo), con ciertas expectativas de continuación.

- Reuniones formales e informales con el profesorado de Apoyo a la Integración.

- Colaboración y asesoramiento en la realización de las ACIs Programa de Diversificación Curricular.

- Preparación del diseño y planificación de una Escuela de Padres para el próximo curso escolar.

Entre las PROPUESTAS de mejora se señalan las siguientes:

- Es necesario que el profesorado (tutores/as) siga implicándose en el diseño, desarrollo y evaluación del Plan de Acción Tutorial.

- Seguir proponiendo desde la Jefatura de Estudios una hora semanal, como se ha realizado hasta ahora para la coordinación con tutores del Primer Ciclo, otra para los tutores de $3^{\circ}$ de ESO y otra para los de $4^{\circ}$ de ESO.

- Se intentará trabajar un Plan contextualizado y adaptado a las necesidades del alumnado, con la posibilidad de ajustar algunos objetivos a la realidad concreta del grupo-clase. 
- Recabar información del nuevo alumnado que se integra por primera vez en el Centro (alumnos de $1^{\circ}$ y $3^{\circ}$ de ESO) sobre:

- Niveles de competencia curricular.

- Desarrollo intelectual.

- Historial académico.

- Historia evolutiva y familiar.

- Intereses y motivaciones.

- Gustos y preferencias profesionales, ...

\section{Tareas con las jefaturas de estudios}

Las tareas de coordinación entre los distintos Jefes de Estudios y el Orientador hicieron posible materializar la planificación de los diferentes programas y actividades, tratando de proporcionar dimensiones espacio-temporales, recursos materiales y conjuntando los esfuerzos comunes.

- Asistencia y colaboración en las distintas sesiones de evaluación y promoción de la ESO.

- Coordinación en la planificación de actividades desarrolladas en el centro por personal especializado y externo al mismo.

- Preparación y selección de materiales referidos a evaluación sobre el proceso de enseñanza y aprendizaje, destinados al profesorado que se incorporó al centro por primera vez.

- Coordinación con las Jefaturas de Estudios de Bachillerato y F.P. para el análisis contextualizado del alumnado de enseñanza Postobligatoria (Orientación en el Bachillerato, estudio sobre el proceso de toma de decisión sobre elección de estudios seguido por el alumnado de postobligatoria antes de formalizar la matrícula en el IES Torreblanca durante el curso 1998/99, ...).

- Continuar las líneas de acción para la elaboración para el Proyecto Curricular de Etapa de Bachillerato.

Entre las PROPUESTAS cabe resaltar:

- Las condiciones, en la medida de lo posible, a tener en cuenta para el funcionamiento y organización de las tutorías:

- La hora de tutoría no debe fijarse dentro de la banda horaria del alumno/a ni al principio ni al final de jornada.

- Organización del alumnado en función de los agrupamientos flexibles y según el nivel de competencia curricular del alumnado. para ello disponemos de información pertinente de los alumnos/as que vienen de Primaria. En $2^{\circ}$ de ESO disponemos de datos, según la última evaluación realizada, todavía susceptibles de sistematizar para la realización de estos posibles agrupamientos. 
- Para ello se precisa una evaluación diagnóstica inicial del alumnado que nos irá permitiendo mover al mismo hasta encontrar un ajuste realista para el desarrollo y evaluación de las programaciones de aula.

- Se trata, pues, de adaptar los programas de aula a las necesidades reales del alumnado, en función de la respuesta educativa, intentando detectar dificultades de aprendizaje que vayan surgiendo durante el mismo proceso de aprendizaje e ir retroalimentando el mismo proceso, siempre con la posibilidad de ir tomando decisiones para que el ajuste sea lo más coherente.

- Individualización aún más del aprendizaje de los alumnos/as con necesidades educativas especiales, llevando a cabo las Adaptaciones Curriculares correspondientes.

\section{Tareas con padres}

\section{Actividades grupales}

- Charlas iniciales a las familias cuyos hijos se integraban por primera vez en el centro: LOGSE, el centro y su infraestructura, espacios y aprovechamiento de los mismos, la ESO: características, materias, Ciclos y niveles,etc.

- Información y asesoramiento a familias de los alumnos/as adscritos al Programa de Diversificación Curricular u otros no adscritos (alumnos de apoyo a la Integración y con graves dificultades en el aprendizaje).

- Información y asesoramiento a las familias cuyos hijos/as estaban a punto de finalizar la etapa de Educación Secundaria Obligatoria, sobre posibles estudios y alternativas de formación dentro y fuera del Sistema Educativo.

\section{Actividades individuales}

- Entrevistas con padres previa solicitud o no, o bien a petición del tutor u orientador (temas personales, familiares, escolares, ...).

- Seguimiento de alumnos/as con necesidades educativas especiales y derivación a otros centros colaboradores para cumplimentar diagnósticos específicos complementarios.

- Seguimiento individualizado con padres de alumnos de Diversificación Curricular o necesidades educativas especiales.

\section{Relaciones con el entorno}

Entre los organismos públicos y privados, asociaciones, fundaciones, etc. que han mantenido relación directa con el Departamento de Orientación, aportando ayuda específica en distintos aspectos, bien con personal especializado o bien con la aportación de materiales para el desarrollo de las actividades planificadas, están:

- Delegación de Educación y Ciencia. 
- Secretariado de Acceso.

- Salón Internacional del Estudiante.

- Ayuntamiento de Sevilla.

- Consejería de Asuntos Sociales.

- Laboratorios Rembrandt (Educación Sexual).

- Universidad de Sevilla.

- Servicios Auxonia (Educación Sexual).

- Fundación FONAT.

- INEM

- Alcohólicos anónimos.

- Centros de otras zonas educativas: visitas.

- Centro de Profesores/as.

- EOE (Equipo de Orientación Educativa) de zona.

- Asociación Garrula.

- Fundación La Caixa.

- Instituto de la Mujer.

- Monitores de Cursos de Formación Ocupacional para el desarrollo de programas de prevención de drogodependencias. Consejería de Trabajo.

- Programa Integra (Fondo Social Europeo/consejería de Educación y Ciencia de la Junta de Andalucía)

- Proyecto a nivel europeo Maid Marian.

\section{Medidas de atención a la diversidad del alumnado}

\section{Apoyo a la integración}

Nuestro centro Desarrolla un Programa de Integración en el que están adscritos alumnos/as con déficit psíquico y sensorial (déficit auditivo) desde hace dos cursos escolares. cabe mencionar en este sentido las ideas que exponemos a continuación:

- Nuestra propuesta se centra en seguir manteniendo la atención individualizada a los alumnos ya detectados con más dificultades de aprendizaje, los alumnos/as nuevos que se incorporen en el Centro y vienen de Primaria para proseguir el desarrollo de las adaptaciones Curriculares pertinentes.

- Diseñar, desarrollar y evaluar las ACIs pertinentes de los alumnos/as con necesidades educativas especiales en la nueva etapa educativa.

- Realizar la evaluaciones psicopedagógicas a quienes lo precisen.

- Elaborar instrumentos específicos de recogida de datos que determinasen de manera explícita los niveles de competencia curricular que alcanzan los alumnos de un curso para el siguiente. 
- La coordinación mantenida entre profesorado, profesora especialista en Pedagogía Terapeútica y el orientador ha hecho posible que el Plan tuviera aceptación, pleno desarrollo y un sistema de evaluación conjunta con la finalidad de buscar siempre propuestas que mejorasen nuestra práctica docente.

\section{Valoración de las adaptaciones curriculares grupales en el desarrollo de las programaciones de aula en las distintas áreas y materias curriculares}

La valoración global y el grado de satisfacción en general que se desprende del profesorado ha sido positiva por:

- Conseguir mayor ajuste de la programación al alumnado.

- Predominancia de los contenidos procedimentales prioritariamente en grupos donde la situación lo requería.

- El grado de participación del alumnado ha sido bueno aunque el de motivación e interés se apreciaba muy desigualmente.

- Ha permitido a los alumnos alcanzar objetivos básicos dentro del ciclo y nivel educativo.

- El grado de motivación aumenta cuando el alumno se siente más protagonista y participa más habitualmente en las tareas escolares.

- El reducido número de alumnos/as en algunos grupos ha permitido llevar a cabo estos planteamientos. No podemos afirmar que la ratio poco elevada sea el factor único y decisivo para una mejora de la calidad de la enseñanza, pero sí bastante influyente en el rendimiento del alumnado al prestarle una atención más individualizada y directa. El interés, motivación, participación en las tareas son elementos también importantes y necesarios para que el proceso de enseñanza-aprendizaje se desarrolle con normalidad.

- La diversidad en la propuesta de actividades influye para una posterior implicación y participación en las mismas.

Es necesario seguir trabajando en esta línea y teniendo en cuenta los siguientes principios educativos:

- Evaluar el nivel de competencia curricular en las diferentes áreas y materias del alumnado en cada nivel.

- Dependiendo de la evolución y el progreso del alumnado llevar a la práctica el refuerzo de los diferentes contenidos conceptuales, actitudinales y procedimentales dentro del nivel en el que se encuentra el alumnado.

- Trabajar el autoconcepto y autoestima en los alumnos/as que presenten un bajo nivel en este sentido. Está demostrada la incidencia directa de este factor en el Rendimiento Académico de los alumnos/as.

- No incluir, en la medida de lo posible, muchos alumnos repetidores dentro de un grupo. 


\section{Evaluación de la tutoría por los tutores/as}

1. Manifestaciones observadas en los alumnos/as:

- Poca participación en algunos casos, en otros no tanto. Desinterés cuando las actividades no conectaban con sus intereses y motivaciones.

- Falta de motivación y trabajo igual que en el resto

de las áreas para grupos de alumnos/as puntualmente señalados.

2. Aspectos mas positivos:

- Respeto y tolerancia entre el alumnado.

- Establecer un clima de cooperación y diálogo, la confianza entre el grupo, la cohesión grupal, la unión.

- Las diferentes actividades diseñadas en consonancia con los intereses y motivaciones propias de su edad.

3. Aspectos mas negativos:

- Falta de trabajo e interés.

- Falta de comunicación con algunos profesores/as.

- Pasividad y relajación en algunos alumnos.

- Mayor seguimiento en alumnos con problemas personales y de actitud.

- A veces es necesario potenciar el respeto a las diferencias y la aceptación del otro.

4. Los tutores hubieran necesitado mas:

- Colaboración y participación de las familias.

- Mayor seguimiento en ciertos alumnos que manifestaban problemas de personalidad, actitudes, ...

- Tiempo para la tutoría (es insuficiente una hora).

- Participación del alumnado.

- Actividades socio-culturales (asistir a obras de teatro, exposiciones, ...).

5. Aspectos favorecedores:

- Desarrollo de un clima adecuado y entendimiento entre los alumnos.

- Empatía Profesor/a tutor/a- alumnos/as.

- Actitud dialogante y abierta que ha favorecido la comunicación.

- Otros aspectos resaltados anteriormente.

6. Aspectos perturbadores:

- Falta de orden.

- El hecho de hacer coincidir la hora de tutoría al final de jornada, cuando el alumnado está influenciado por un número de estímulos considerable.

- Cierta apatía por parte de un número determinado de alumnos/as y, todos los aspectos negativos que aparecen en el apartado $n^{\circ} 2$. 


\section{Propuestas de mejora}

$1^{\circ}$ Trabajar temas como:

- Disciplina en el aula.

- Planificación del estudio

- La motivación a través de temas seleccionados por los alumnos/as.

- Otros.

$2^{\circ}$ Posibilidad de trabajar la tutoría con menos alumnos/as o hacer la tutoría compartida para obtener un mayor conocimiento del alumnado y de sus problemáticas con la finalidad de ayudarles personal, escolar y vocacionalmente.

$3^{\circ}$ Consensuar a nivel de grupo las temáticas de trabajo por cada trimestre, y parte de las actividades comunes de obligada realización (cuestionarios iniciales, preparación de las evaluaciones, elecciones de Delegado, ...).

$4^{\text {o }}$ Trabajar los valores y actitudes positivas en el alumnado y de desarrollo personal: respeto, tolerancia, diálogo, maltrato, discriminación, autoconcepto, autoestima, motivación, ...

$5^{\circ}$ Conseguir motivar a los alumnos/as para su mayor participación en las tareas.

\section{Programa de diversificación curricular. Valoración global}

\section{Valoración por el profesorado}

\section{Resultados en los ámbito socio-lingüístico y científico-tecnológico}

En líneas generales, puede decirse que el desarrollo de la programación en estos ámbitos se ha llevado a cabo de forma regular pero con un rendimiento académico más bajo que el curso anterior debido a los niveles de competencia curricular que presentaban los alumnos adscritos al programa.

Como se ha comprobado desde que se inició el programa, este planteamiento supone bastantes ventajas para su desarrollo, pues los alumnos se sienten más motivados; no se distraen con la facilidad que pueden tener en un curso más numeroso con compañeros faltos de interés y el profesor puede hacer todas las adaptaciones personales que necesiten. La participación de todo el alumnado está asegurada de un modo habitual.

El programa estaba compuesto por trece alumnos/as de los cuales uno de ellos presentaba un déficit psíquico y dos con déficit sensorial (déficit auditivo). El abandono que se produce por parte del alumnado viene ocasionado sobre todo por problemas personales y familiares cuya incidencia directa se reflejaba en la falta de interés y compromiso por las tareas escolares, a pesar de los intentos por motivarles y potenciar el autoconcepto y autoestima.

El resto del alumnado ha mostrado bastante interés y participación, realizando las tareas en mayor o menor medida (uno de ellos trabajaba el resto del día), aunque en algún caso, a 
veces, se dejaban influir por el compañero cuya actitud hemos reseñado más arriba de forma negativa.

Como en cursos anteriores, a excepción de un alumno en Lengua y otro en Geografía e Historia, pueden obtener el título de ESO por haber cubierto básicamente los objetivos, y dos de ellos lo han cubierto bastante satisfactoriamente.

No obstante, siempre puede mejorarse la programación planteada y sería necesario conseguir materiales más idóneos para llevarla a cabo, pues hay que adaptarlos a cada alumno y a veces es escasa la dotación o, en algunos casos, no están todavía suficientemente adaptados.

\section{Currículum común}

La dificultad mayor encontrada es el número de alumnos tan elevado en el grupo que impide la atención individualizada del alumnado de diversificación. No se puede atender a éste del mismo modo que en el currículum específico.

Al adaptar algunos elementos del currículum a las características de los alumnos tienen la posibilidad de cooperar, participar y obtener así, el Título de ESO.

En general, el grado de adaptación del alumnado es alto, salvo algunos casos excepcionales que han mostrado cierta actitud relajada y pasiva.

No se observa trato discriminatorio ni entre los mismos alumnos ni por parte del profesorado.

El grado de motivación, interés, participación, realización de tareas y trabajos ha sido buena aunque iba aumentando conforme avanzaba el curso. La asistencia ha sido alta, salvo en casos aislados y en los casos en que se produjo abandono -según se ha dicho anteriormente-.

El número de alumnos evaluados satisfactoriamente es de 10; 2 insatisfactoriamente y uno abandona.

\section{Propuestas de mejora}

- Reducir el número de alumnos en las materias del currículum común.

- Mantener entrevistas personales entre profesores y alumnos.

- Trabajar la autoestima, el autoconcepto, seguridad en sí mismo,etc. de modo inherente al quehacer educativo de la vida escolar.

- Reducir las posibles discrepancias en cuanto a la evaluación y el rendimiento académico de los alumnos/as en el currículum común (rendimiento más bajo) y el currículum específico (adaptaciones curriculares totalmente aseguradas, metodología distinta, ...).

\section{Evaluación de la tutoría con el orientador}

Se puede decir que se han alcanzado los objetivos previstos, siendo la valoración global de la tutoría bastante positiva y satisfactoria. 
El grado de implicación y participación en las tareas desarrolladas ha sido bastante alto, debido a la propia motivación que suscitaba en el alumnado la propuesta de actividades que se iban planteando.

Los bloques de contenido y temáticas tratadas se pueden resumir de la siguiente manera:

- Integración del alumnado en el grupo.

- Conocimiento de sí mismo y el grupo.

- Autoconcepto y autoestima.

- Orientación vocacional y profesional: programa ELIGE, cuyos ámbitos de contenido fueron:

- Conocimiento de sí mismo.

- Conocimiento del mundo del trabajo.

- Información académica.

- Salidas profesionales.

- Toma de decisiones.

- La perspectiva de género dentro del programa.

- Técnicas sobre la Búsqueda de Empleo.

- Resultados académicos obtenidos:

PROMOCIONAN CON TITULO DE LA ESO

10 ALUMNOS

NO PROMOCIONAN (CERTIFICACIÓN)

2 ALUMNOS

ABANDONOS

1 ALUMNO

- Propuesta de adscripción al nuevo programa:

13 alumnos/as para el curso próximo 1998/99

\section{Evaluación del programa por el alumnado}

No encuentran grandes dificultades en cuanto al aprendizaje del alumnado, aunque de modo aislado en las áreas de Lengua Castellana, Plástica y Visual y Educación Física.

Entre las ventajas que encuentran los alumnos/as están:

- «Todos los profesores ayudan y explican con más detenimiento.

- Han aprendido más.

- Al estar menos alumnos, las clases se pueden dar mejor.

- Hay más posibilidades de aprobar.

- Se explica mejor y con más facilidad.

- Se enseña de un modo diferente y más adecuado.

- El profesorado controla y sigue el aprendizaje del alumnado mejor. 
- La participación es mayor.

- El profesorado nos escucha y comprende mejor.»

Son ideas y respuestas recogidas de las diferentes técnicas e instrumentos aplicados para evaluar el programa.

Entre las propuestas de mejora señalan ideas recogidas entre las diferentes técnicas aplicadas:

- «Poner solamente el primer idioma y de un modo voluntario para los alumnos/as.

- Aula más grande.

- Está bien tal y como está.»

Los alumnos/as se han adaptado al programa, excepto tres alumnos (dos alumnos y una alumna), igual que los profesores también se han adaptado a los alumnos/as, ayudándoles en la medida de lo posible.

La valoración global del programa se puede calificar como muy buena, exceptuando estos casos aislados de alumnos no adaptados. En general esta es la idea generalizada que tenemos en nuestro contexto educativo desde que el curso escolar 1993/94 se empezaron a experimentar estos planteamientos curriculares como última medida de atención a la diversidad del alumnado dentro del actual y nuevo Sistema Educativo.

Las materias que han resultado más motivadoras para ellos han sido Lengua Castellana, Ciencias de la Naturaleza, Matemáticas y en menor medida Ciencias Sociales. De igual modo estas son las materias en las que más conocimientos han adquirido. Es la metodología el elemento curricular más resaltado por los alumnos/as, destacando los aspectos procedimentales a lo largo del desarrollo de los diferentes contenidos curriculares.

En cuanto a la percepción del trato discriminatorio se ha percibido en muy poca medida en algunos profesores y alumnos del grupo ordinario; a veces se tomaban decisiones en el grupo sin contar con la opinión del alumnado de Diversificación Curricular. En general no se han percibido reacciones o trato discriminatorios hacia el alumnado de D.C.

Sobre las expectativas que ellos mismos tenían antes de ser evaluados finalmente eran las siguientes:

- Merecen obtener el título en ESO 9 Alumnos:

Las razones que argumentan son:

- «Esfuerzo realizado.

- Tiempo dedicado al estudio.

- Se ha trabajado lo suficiente.

- El Grado de participación ha sido alto».

- Dos alumnos no contestan.

- Un alumno no merece la obtención del título.

La valoración que conceden a las distintas materias: metodología, actividades, temporalización, grado de motivación, aprendizaje, ...) es la siguiente: 


\begin{tabular}{|l|c|}
\hline \multicolumn{1}{|c|}{ Materias } & Valoración \\
\hline Ámbito científico-tecnológico & 7,4 \\
\hline Ámbito socio-lingüístico & 7,8 \\
\hline Educación física & 5,8 \\
\hline Informática & 7,5 \\
\hline Religión & 5 \\
\hline Alternativa a la religión & 5,5 \\
\hline Vida moral y reflexión ética & 5,5 \\
\hline Educación plástica y visual & 5,8 \\
\hline Tutoría con tutor & 6,6 \\
\hline Tutoría con orientador & 7,5 \\
\hline
\end{tabular}

\section{Consideraciones finales}

El pleno desarrollo de las funciones, tareas, programas y actividades propuestas desde el Departamento de Orientación ha sido posible gracias a la dinamización que éste ha establecido en el centro desde principios de su actuación en el curso escolar 1992/93; a la participación y colaboración del profesorado que nunca miró con recelo las funciones y tareas relacionadas con la orientación; a la colaboración, aceptación y agradecimiento por parte de las familias y los mismos alumnos; al respaldo y apoyo constante de las personas encargadas de gestionar el centro durante este período de tiempo (Equipo Directivo), siendo éste también dinamizador en cuantos procesos de cambio se iban introduciendo en el contexto educativo y sirviendo de clave importante para la viabilidad y efecto de las diferentes propuestas de intervención que se pretendían planificar, implementar y evaluar.

Aún así, somos conscientes de que la administración educativa, siendo conocedora de la organización y funcionamiento de estos departamentos, debieran arbitrar medidas, explícitas en normativa legal sobre orientación, más contundentes y definitorias encaminadas a subsanar ciertas deficiencias y carencias que venimos observando desde que comenzamos por estas andanzas de la orientación dentro de un marco institucional: por destacar algunas de ellas, está la voz reiteradas entre los que ejercen la función de tutor de potenciar esta figura, reduciendo el número de horas lectivas; aumentando el número de orientadores por centro según ratio y etapas educativas que se imparten en el mismo; incentivar los apoyos mediante profesorado perteneciente a este Departamento, planteamiento obvio en cualquier contexto educativo; la existencia de medios materiales; coordinación con diferentes entidades y organismos oficiales (Consejería de Asuntos Sociales, Consejería de Trabajo, INEM, ...); conexión a Internet, que facilitase la actualización académica y profesional del alumnado; en definitiva, una evaluación real y objetiva de estos departamentos realizada con personal externo al propio centro, con vistas a mejorar su organización y funcionamiento, paliar las deficiencias, potenciar su dinamización y conseguir una verdadera orientación de calidad. 
Los servicios de orientación educativa constituyen un elemento esencial a la hora de garantizar la compensación de las desigualdades y la atención a la diversidad del alumnado que pudiera estar integrado en un centro determinado. Por ello, y esperando en los albores del siglo XXI esta garantía se consolide y se haga realidad una orientación bien planificada en nuestro actual sistema educativo.

Fecha de recepción: 26-6-00

Fecha de revisión: 15-9-00

Fecha de aceptación: 21-11-00 